\title{
Efectos que producen el KCl y el isopropanol a la pectina en solución en la resistencia al flux en membranas de ultrafiltración
}

\author{
Antonio Hidalgo Millán \\ Área de Ciencias Básicas e Ingenierías, Universidad Autónoma de Nayarit, Ciudad de la Cultura "Amado Nervo", Tepic, Nayarit, \\ México CP 63155. \\ E-mail:anhimi@gmail.com
}

Recibido 7 de enero de 2013, Aceptado 1 de febrero de 2013

\begin{abstract}
Resumen
La Ultrafiltración de la pectina en solución en régimen laminar ha sido experimentalmente estudiando utilizando perturbaciones iónica $(\mathrm{KCl})$ y por solvente (isopropanol). Los cambios inducidos por estos compuestos fueron evaluados por los métodos siguientes: a) Análisis visual, b) Reología de cizalla simple; y c) Resistencia al flujo a través de las membranas durante el proceso de ultrafiltración. El análisis visual permitió distinguir 6 diferentes morfologías en las soluciones de pectina y cuando se procesaron mediante ultrafiltración, el flux a través de las membranas disminuyó rápidamente, estabilizándose a valores bajos y presentando una muy alta resistencia especifica. Los cambios en la reología fueron evidentes al pasar de fluido newtoniano a uno no-newtoniano en las soluciones pretratadas con isopropanol. Un comportamiento similar se observó en las soluciones de pectina concentradas por ultrafiltración.
\end{abstract}

Palabras Clave: Ultrafiltración, Fuerza Iónica, Pectina, Reología, flux de Permeado.

\begin{abstract}
Ultrafiltration of Pectin in solution in the laminar regimen has been experimentally studied using Ionic strength $(\mathrm{KCl})$ and solvent perturbation (Isoprapanol). The changes induced by these components were evaluated by the following methods: a) Visual analysis; b) Steady shear rheometry and; c) Strength to flux through the membrane during the ultrafiltration process. Six different morphologies of pectin solutions were distinguished by visual analysis. When processing by ultrafiltration the solutions, the flux through the membranes decreased quickly by stabilizing at low values and very high membrane specific strength were obtained. A rheological change was observed from a Newtonian behavior to a nonNewtonian for the solutions pretreated with isopropanol. A similar behavior was observed when the solutions of pectin were concentrated by ultrafiltration.
\end{abstract}

\section{Introducción}

La filtración está definida como un proceso de separación de dos o más componentes de un fluido, basado principalmente en la diferencia de tamaño de partículas. La ultrafiltración utiliza este mismo principio, por lo cual se puede decir que la ultrafiltración es una filtración con algunas variantes en su operación. Por otra parte, las cada vez más exigentes normas de impacto ambiental en los procesos industriales actuales, han llevado a la investigación y desarrollo de nuevos procesos biológicos cada vez más limpios y que cumplan con las normas actualmente establecidas. En este sentido, los procesos de separación mediante una membrana semipermeable como en la ultrafiltración (UF), han sido considerados como una alternativa real y con un alto grado de desarrollo en una amplia variedad de separaciones biotecnológicas $[1,2]$, esto gracias a sus moderadas condiciones de operación (temperatura y presión bajas) y en general por su bajo consumo de energía (aunque este factor depende mucho de la geometría de las membranas). Uno de los factores más importantes es el hecho de que la UF no somete a la solución a un cambio de fase, como en el caso de la evaporación. Sin embargo, en la UF se llegan a tener el problema de una disminución importante en el flux de las membranas, resultando en un decremento costoso del rendimiento del proceso [3, 4]. Este fenómeno es atribuido a dos causas principales; la primera es la adsorción de macromoléculas asociada a una interacción específica entre las macromoléculas y la superficie de la membrana polimérica en el interior de sus poros y, la segunda es la llamada polarización de la concentración, la cual se refiere a la acumulación de macromoléculas en la superficie de la membrana, llegando a formar una torta en forma de gel sobre su superficie, ocasionando una resistencia adicional al paso del fluido a través de la membrana [5]. Muchos autores han reportado diversas estrategias para lograr disminuir este par de fenómenos presentes en la operación de 
ultrafiltración. Entre las más importantes, se pueden mencionar a las tres principales: 1) $\mathrm{La}$ optimización de las condiciones hidrodinámicas del proceso, 2) La modificación en el diseño de los módulos de la membrana (en ambos casos, con el propósito de lograr un mayor grado de turbulencia e incrementar en gran medida el coeficiente de transferencia de masa) y 3) El desarrollo de nuevas y mejores membranas, mejorando la distribución y el tamaño de los poros, disminuyendo la cantidad de poros que permitan un paso de partículas de tamaño mayor al deseado, o bien creando membranas hidrofílicas para minimizar el efecto de la interacción con las soluciones acuosas $[3,6]$. Tal es el caso de la microfiltración de jugo de manzana [7], BSA [8], soluciones de xantana [9], IgY [10]. Con esta estrategia se logró relacionar patrones coloidales de agregación con la resistencia al flux de la película formada en la superficie de la membrana, logrando aumentar hasta en dos órdenes de magnitud el flux en la membrana. Estos resultados sentaron las bases para seleccionar, de una manera racional, los pretratamientos utilizando la fuerza iónica y el agregado de solventes para el procesamiento de estos productos. Sin embargo, los resultados de éstos estudios no pueden ser extrapolados a otros sistemas macromoleculares, debido a la falta de teorías que expliquen la dependencia de estructuras coloidales a variables como fuerza iónica y reacción con solventes, así como tampoco se han establecido teorías que expliquen la dependencia de dichas estructuras a modelos de predicción de flux. Es por ello que es necesario realizar un estudio para la pectina en solución, con la finalidad de determinar su patrón de comportamiento, al someter las soluciones a los pretratamientos aquí propuestos. La pectina es un polisacárido de unidades de ácido galacturónico presente en las plantas superiores $[11,12]$ y son importantes por su enorme poder gelante en concentraciones inferiores al $1 \%$ en peso. Estas pueden provocar la gelación de jugos de frutas, formando jaleas $[13,14]$ y esta acción, junto a su capacidad para actuar como agentes emulsionantes, ha dado lugar a su producción comercial a gran escala.

Las alternativas anteriormente expuestas para mejorar el proceso de ultrafiltración, han sido la principal inquietud para la realización de este estudio a partir de perturbaciones estructurales a la macromolécula de pectina en solución, ocasionadas por efectos de fuerza iónica y de adición de solventes que disminuyan la resistencia al flux de la membrana y favorezca el rendimiento del proceso. Estos cambios se identificaron mediante observación visual y reometría de cizalla simple.

\section{Desarrollo experimental}

\subsection{Preparación de las soluciones}

La preparación de las soluciones de pectina (GENU PECTIN, PKelco Food Gums Division, USA) de 0.5 a $2.0 \% \mathrm{p} / \mathrm{v}$ se realizaron disolviendo el biopolímero en agua. La disolución de la pectina en polvo, se llevó a cabo en un agitador magnético a una velocidad de $400 \mathrm{rpm}$ durante un período de tiempo entre 2 y 4 horas (dependiendo de la concentración), a temperatura ambiente (aproximadamente $25{ }^{\circ} \mathrm{C}$ ). La pectina fue adicionada lentamente, para evitar la formación de grumos. A su vez, se les adicionó azida de sodio (200ppm) para su conservación. Con la misma metodología de preparación se realizaron las soluciones de 0.5 a $1.25 \% \mathrm{p} / \mathrm{v}$ de pectina con 0.5 $1.25 \% \mathrm{p} / \mathrm{v}$ de $\mathrm{KCl}$, las de $0.5-1.25 \% \mathrm{p} / \mathrm{v}$ de pectina y $20-50 \% \mathrm{v} / \mathrm{v}$ de isopopanol y las de pectina (0.5$1.25 \% \mathrm{p} / \mathrm{v}), \mathrm{KCl}(0.5-1.25 \% \mathrm{p} / \mathrm{v})$ e isopropanol $(20-50 \% \mathrm{v} / \mathrm{v})$, en todas las combinaciones posibles que pudieran ser tratadas por ultrafiltración: En todas las soluciones, inicialmente se disolvió la pectina, después se adicionó el $\mathrm{KCl}$ y por último se agregó el isopropanol, todo esto en un tiempo máximo de 8 horas. Para todas las soluciones se prepararon al menos dos lotes y se realizaron pruebas por triplicado para cada uno de ellos, para comprobar la reproducibilidad de los resultados y se almacenaron a $4{ }^{\circ} \mathrm{C}$ durante 24 horas, para alcanzar un estado estable en la solución, previo a su uso o análisis.

\subsection{Descripción visual}

Previa utilización de las muestras, se realizó el análisis visual de las soluciones, de acuerdo al procedimiento reportado en la literatura [15]. Se definió la morfología de las soluciones en base a lo observado por dos personas, tomando como criterios: viscosidad, opacidad, gelificación, agregación y precipitación (Fig. IV-1). Los niveles establecidos para criterio fueron: ligeramente, moderadamente y altamente. La descripción de un gel, describe que un gel debe permanecer elástico y tener capacidad de recuperación al tacto $\mathrm{y}$, sobre unos segundos no debe exhibir flujo bajo su propio peso. El aspecto de las soluciones observadas fue agrupado y clasificado de acuerdo a estas características.

\subsection{Determinaciones Reológicas}

Se evaluaron las propiedades reológicas de las 
soluciones de pectina en un reómetro rotacional (Haake, Germany, Mod. CV20N) usando una geometría de cilindros concéntricos (ZB15, distancia entre cilindros $=1 \mathrm{~mm}$ ) y una temperatura controlada por un baño de agua (Haake, Germany, Mod. F3T) a $25^{\circ} \mathrm{C}$. Para estimar la manera en que los cambios conformacionales en la molécula de pectina, inducidos por el $\mathrm{KCl}$ y el Isopropanol, influían en la viscosidad de las soluciones. . Los valores reportados, son el promedio de tres réplicas, a la misma concentración y con soluciones diferentes.

\subsection{Experimentos de Ultrafiltración}

Los experimentos de ultrafiltración se realizaron en un equipo de un solo canal con flujo tangencial (Figura 1) (Amicon, Bioseparations, Millipore, Mod. Pellicon XL Biomax 10). El equipo está conformado por una bomba peristáltica de diferentes velocidades, longitud del canal $=7.4$ pulgadas $(188 \mathrm{~mm})$, ancho del canal $=1.2$ pulgadas $(30.48 \mathrm{~mm})$, altura del canal $=0.38 \mathrm{~mm}$, el área de filtración efectiva de la membrana fue de $0.005 \mathrm{~m} 2$ y la presión máxima de operación fue de 60 psia. Se utilizó un recipiente contenedor de acrílico para la solución a tratar, con una capacidad de $2000 \mathrm{ml}$, (mostrado en la Figura 1). La presión en la membrana era controlada mediante una perilla que cerraba o abría el flujo de la solución a la salida de la membrana y era medida con dos barómetros colocados uno anterior a la entrada y el otro posterior a la salida de la membrana. Las determinaciones del flux de permeación se realizaron con una membrana de polisulfuna de 30 $\mathrm{KDa}$, operando a flujo tangencial. Se hicieron dos tipos de experimentos, los primeros a concentración constante (adicionando el permeado en el concentrado cada $10 \mathrm{ml}$ ) y posteriormente concentración variable (el permeado que se obtenía no era regresado al tanque contenedor de la solución). En ambos casos, la presión de operación de la membrana fue de 20 psia. Todas las determinaciones fueron hechas por duplicado y el resultado registrado es el promedio de dichas mediciones (con un error de confianza de $+/-5 \%$ ).

\subsection{Limpieza de las membranas}

Previo a cada corrida experimental, la membrana fue lavada hasta obtener el mismo flux inicial, para asegurar la obtención de datos y resultados sujetos a las mismas condiciones. Esta, se colocó en el equipo de ultrafiltración y se pasó un flujo inicial de agua destilada (500 ml para cada lavada). Posteriormente se limpió con una solución de $\mathrm{NaOH} 0.1 \mathrm{M}$, durante 45 minutos (siguiendo las

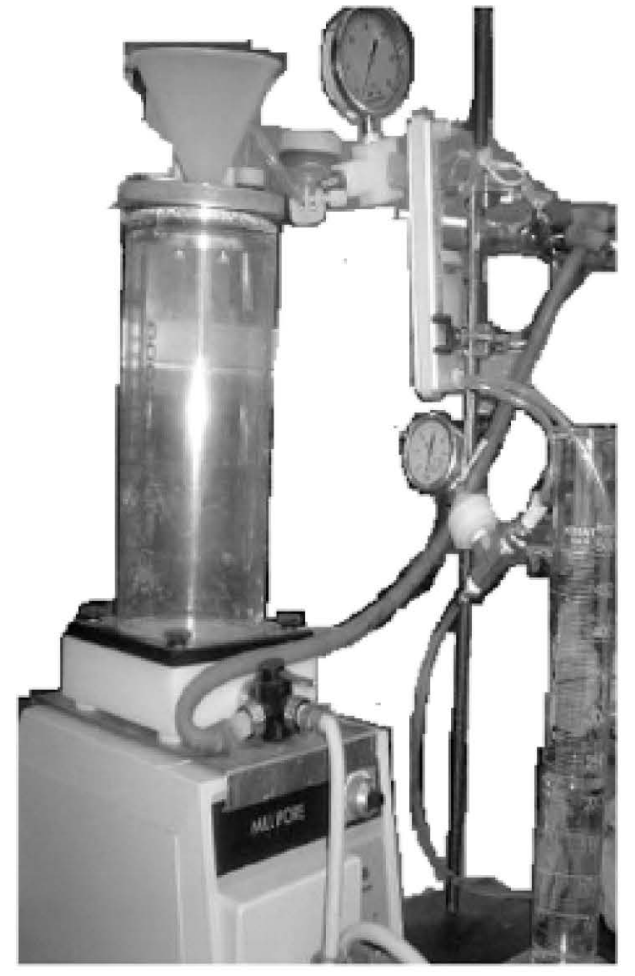

Figura 1. Sistema de ultrafiltración tangencial (Amicon, Bioseparations, Millipore, Mod. Pellicon XL Biomax 10).

instrucciones del manual de operación de la membrana), se continuó su lavado con agua desionizada $(500 \mathrm{ml})$ en dos ocasiones, para eliminar todos los residuos presentes de la solución limpiadora y se procedió a determinar su flux con agua desionizada. Una vez recuperado el flux inicial, se procedía a realizar los experimentos de ultrafiltración. El tiempo de filtración fue medido utilizando un cronómetro. Se cuidó que las mangueras quedaran suspendidas libremente sin ningún tipo de torcedura o ahorcamiento, para evitar la generación de una resistencia adicional al sistema.

\section{Resultados y discusiones}

\subsection{Caracterización visual}

A partir del análisis visual se encontraron seis diferentes morfologías para la totalidad de las soluciones sujetas a estudio. Los criterios seguidos para describir los cambios en su apariencia, se resumen en la Tabla 1. 
Tabla 1. Criterios de descripción de cambios visuales en las soluciones de pectina.

\begin{tabular}{|c|c|}
\hline Morfología & Descripción \\
\hline I & $\begin{array}{l}\text { Líquido ligero a moderadamente viscoso } \\
\text { transparente } u \text { opaco }\end{array}$ \\
\hline II & Líquido altamente viscoso con apariencia de gel \\
\hline III & $\begin{array}{l}\text { Gel opaco. La opacidad aumenta conforme } \\
\text { aumenta la concentración de pectina }\end{array}$ \\
\hline IV & $\begin{array}{c}\text { Líquido opaco viscoso con apariencia de gel y } \\
\text { agregación incipiente }\end{array}$ \\
\hline $\mathrm{V}$ & $\begin{array}{l}\text { Fase superior gel opaco y fase inferior líquido } \\
\text { transparente con agregados }\end{array}$ \\
\hline VI & $\begin{array}{l}\text { Fase líquido transparente con agregados } \\
\text { blancos }\end{array}$ \\
\hline
\end{tabular}

En la Figura 2 se muestran resultados representativos de las morfologías de las soluciones, en ella se observan diferentes apariencias las cuales varían en función de su composición y concentración. La viscosidad y opacidad se incrementó en función de la concentración de Pectina, a mayor concentración mayor turbiedad, esto se tornó más evidente a partir de una concentración de $1.0 \% \mathrm{p} / \mathrm{v}$ del soluto. En el caso de las soluciones de Pectina-KCl, se observaron únicamente morfologías tipo I, lo que evidencia que la adición de la sal provoca variaciones casi imperceptibles [16]. Para las soluciones Pectina-Isopropanol, se observó que su apariencia oscilo entre un rango de tipo I a tipo III, en función al aumento en la concentración del alcohol, sin embargo, el nivel de turbidez no sufrió un incremento sustancial, pero se detectó la aparición de un gel. La visualización del gel se alcanzó a una concentración de $1.0 \%$ de pectina y $40 \%$ de isopropanol.

En general, en la Figura 2 se resumen los resultados obtenidos con el tratamiento estudiado en el aspecto de las soluciones. En ella se observa que la adición de $\mathrm{KCl}$ e Isopropanol, producen los cambios físicos más importantes, los cuales se acentúan a partir de concentraciones del polisacárido iguales o mayores a $0.75 \% \mathrm{p} / \mathrm{v}$. Los cambios ocurridos en las soluciones comprendidas entre estos intervalos, abarcan la mayoría de las morfologías descritas en el presente trabajo. Este hecho puede explicarse debido a que a mayores concentraciones de pectina se obtienen soluciones con formación de un gel más fuerte [17] y al adicionar el alcohol se produce la precipitación de la pectina de manera rápida y efectiva favoreciendo su separación [18].

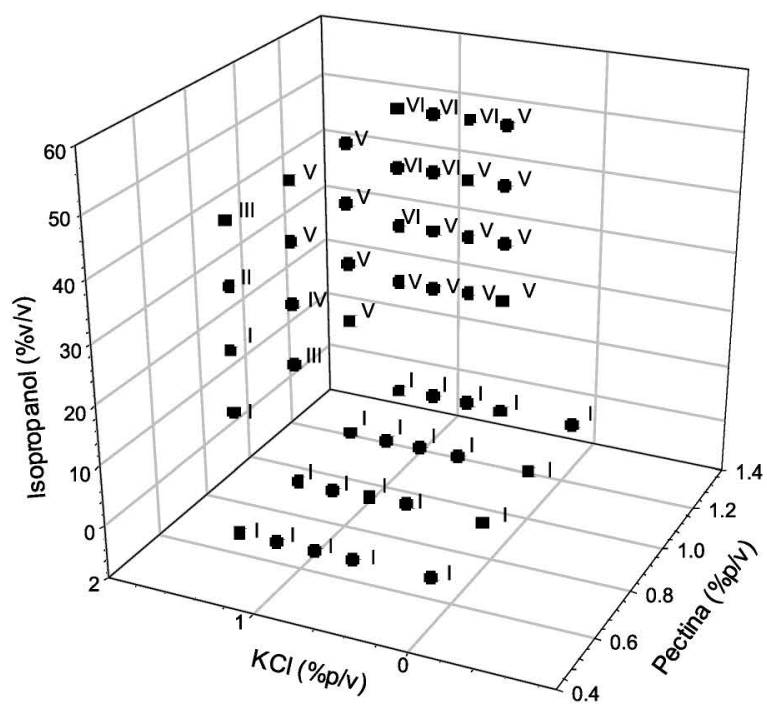

Figura 2. Morfología de las soluciones de pectina con $\mathrm{KCl}$ y/o isopropanol.

La presencia de las diversas morfologías en las soluciones de pectina inducidas por la adición combinada de $\mathrm{KCl}$ e isopropanol se pueden atribuir al cambio de afinidad entre el polímero y el solvente. A bajas concentraciones de alcohol (menores a $30 \% \mathrm{v} / \mathrm{v}$ ) existe un alto grado de afinidad, la pectina es soluble y la adición de la sal no induce un efecto morfológico significativo. Por la naturaleza del polisacárido, se sugiere que bajo éstas condiciones, la molécula se encuentra altamente cargada y se estabiliza a través de interacciones, del tipo enlaces de hidrógeno con agua y del catión con la sal. Esto puede ser atribuido al fenómeno de semiflexibilidad que presenta esta molécula y que tiende a cambiar su conformación estructural [13].

\subsection{Reología de las soluciones}

Se realizaron pruebas de reología de cizalla simple para estimar cambios en la viscosidad de las soluciones, inducidos por la presencia de las perturbaciones estudiadas. Los resultados obtenidos se observan en las Figuras 3 y 4. En la Figura 3 se observa que la viscosidad de las soluciones presenta un comportamiento Newtoniano, debido a que no existe variación en su valor al incrementar la velocidad de corte. Por otro lado, en la Figura 4 se observa que esta tendencia en la viscosidad sufre cambios, a mayores concentraciones del biopolímero; observando que las soluciones concentradas hasta $4 \% \mathrm{p} / \mathrm{v}$, presentan un comportamiento de fluido Newtoniano. Sin embargo, cuando se incrementa la concentración a 
valores mayores o iguales a $5 \% \mathrm{p} / \mathrm{v}$, la viscosidad de las soluciones disminuye a mayores velocidades de cizalla, situación típica de un fluido noNewtoniano con comportamiento pseudoplástico. Los resultados mostrados, son el promedio de 3 diferentes experimentos utilizando la misma solución.

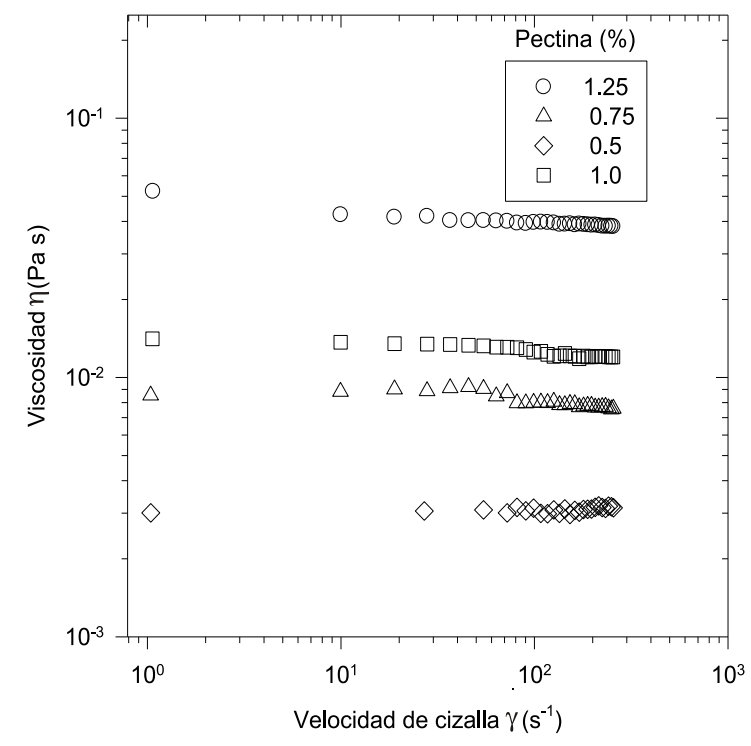

Figura 3. Viscosidad de la pectina en solución a las concentraciones estudiadas

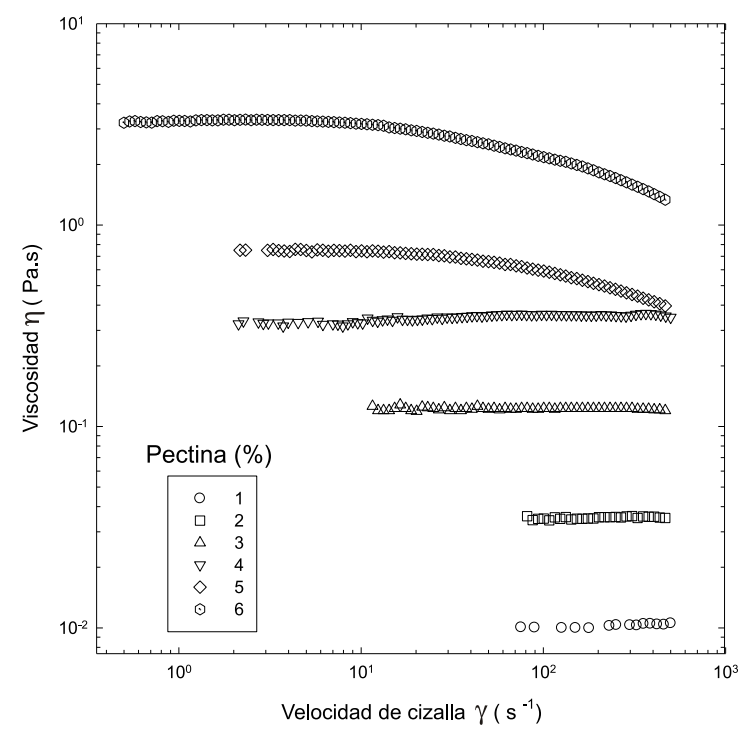

Figura 4. Viscosidad de la Pectina en solución a diferentes concentraciones.
En los fluidos no-Newtonianos, se puede predecir el valor de la viscosidad de la solución a determinada velocidad de cizalla, con la ayuda de un modelo reológico. En nuestro caso, el modelo de Cross es el que mejor se ajusta a los datos experimentales obtenidos, alcanzando el más alto coeficiente de correlación $r^{2}$. Se probaron 2 modelos empíricos, el de la Ley de la potencia y el de Cross de tres parámetros debido a su sencillez y baja complejidad, comparados con modelos empíricos que utilizan un mayor número de parámetros. Los resultados obtenidos se muestran en la Tabla 2.

El modelo de Ley de la Potencia está representado por la ecuación 1 :

$$
\eta=K \gamma^{n-1}
$$

El modelo de Cross de tres parámetros para la viscosidad está dado por la ecuación 2 :

$$
\eta=\frac{\eta_{0}}{1+K \gamma^{n}}
$$

donde: $\eta=$ viscosidad, $\eta_{0}=$ valor de viscosidad inicial estimado (empírico), $K=$ constante característica de la solución, $\mathrm{n}=$ índice de adelgazamiento o de flujo

El ajuste obtenido con la ecuación de Cross de tres parámetros se observa en la Figura 5 y 6 para la solución de pectina al 5 y $6 \% \mathrm{p} / \mathrm{v}$, respectivamente. Los resultados de esta ecuación se muestran en la Tabla 2.

Tabla 2. Reología de soluciones de Pectina no-

\begin{tabular}{|c|c|c|c|}
\hline \multirow{2}{*}{$\begin{array}{c}\text { Solución } \\
\text { acuosa } \\
\text { Pectina } \\
(\% \text { p/v }) \\
\end{array}$} & \multicolumn{2}{|c|}{$\begin{array}{l}\text { Coeficiente de } \\
\text { correlación }\left(\mathrm{r}^{2}\right)\end{array}$} & \multirow{2}{*}{$\begin{array}{c}\text { Ecuación } \\
\text { ajustada } \\
\text { Ley de Cross }\end{array}$} \\
\hline & $\begin{array}{l}\text { Ley de la } \\
\text { Potencia }\end{array}$ & $\begin{array}{c}\text { Ley de } \\
\text { Cross }\end{array}$ & \\
\hline 5 & 0.84 & 0.99 & $\eta=\frac{0.733}{1+0.0081 \gamma^{0.783}}$ \\
\hline 6 & 0.85 & 0.99 & $\eta=\frac{3.4091}{1+0.0188 \gamma^{0.7255}}$ \\
\hline
\end{tabular}
Newtonianas

Estas ecuaciones, obtenidas a partir de los ajustes realizados con el modelo de Cross, nos facilitan la realización de la estimación de la viscosidad de estas soluciones.

La reología de soluciones de pectina ha sido estudiada por diversos autores [18, 19], en sus 
resultados reportan el ajuste del comportamiento no-Newtoniano utilizando el modelo de la ley de la potencia. En sus investigaciones no se hace mención sobre un intento de ajuste con modelos reológicos más complejos como el utilizado en este estudio, lo que dificulta saber si el mejor ajuste de sus datos, era utilizando el de la Ley de Cross de tres parámetros.

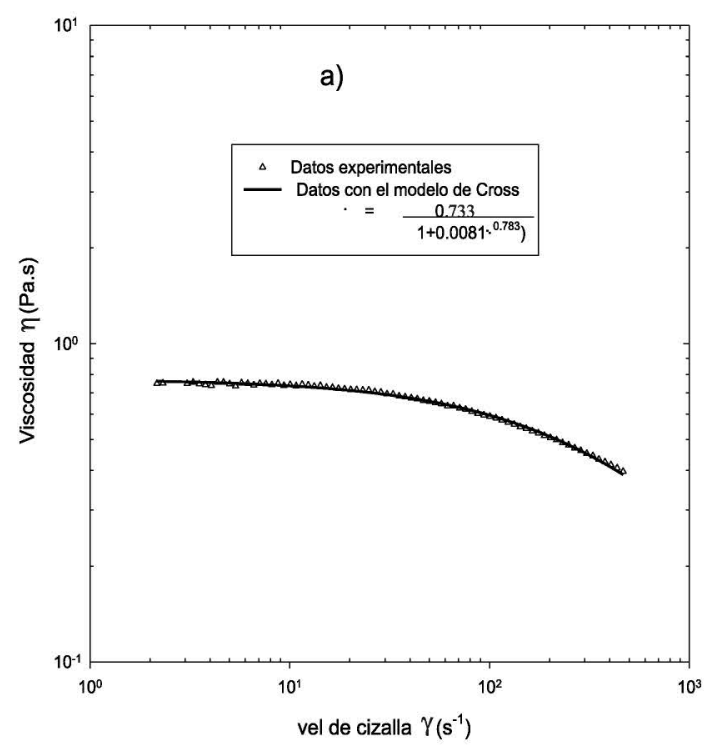

Figura 5. Reograma de una solución de pectina al $5 \%$ $\mathrm{p} / \mathrm{v}$ ajustada con el modelo de Cross.

b)

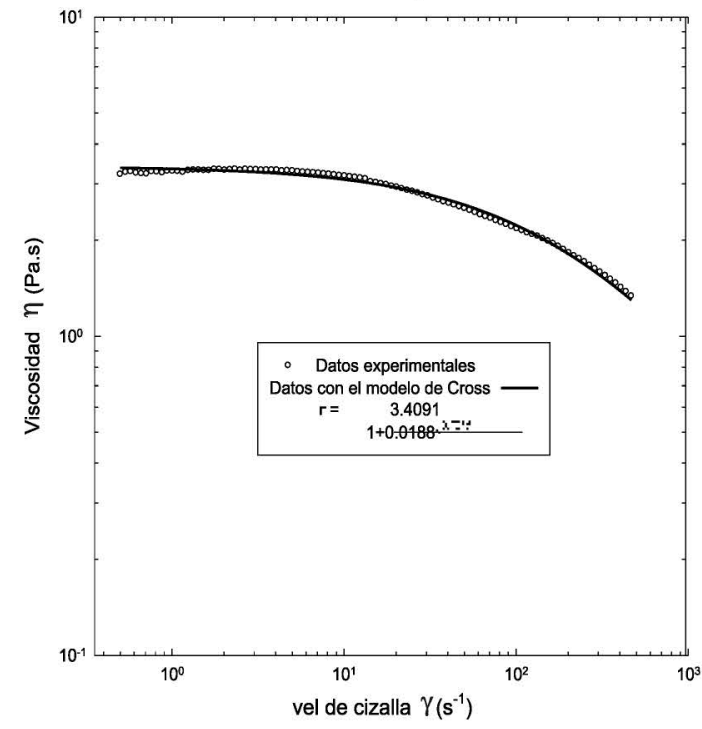

Figura 6. Reograma de una solución de pectina al $6 \%$ $\mathrm{p} / \mathrm{v}$ ajustada con el modelo de Cross.

\subsection{Efecto de las perturbaciones generadas por el KCl y el Isopropanol}

El estudio de las soluciones de pectina perturbadas por fuerza iónica (adición de $\mathrm{KCl}$ ) se observa en la Figura 7. En estas prevalece el comportamiento newtoniano, sin embargo, se aprecia una disminución de la viscosidad con el aumento en la concentración de la sal, situación que modifica la respuesta obtenida por las disoluciones anteriores.

Esta disminución en la viscosidad representa un menor gasto de bombeo de las soluciones tratadas con $\mathrm{KCl}$, debido a que la viscosidad es directamente proporcional a la cantidad de energía que se requiere para poder mover un fluido. Cuando la viscosidad es baja, el deslizamiento de la bomba será mucho mayor al que se tiene con un líquido más viscoso, lo que reduce la capacidad nominal de la bomba y la energía que consume. Estos resultados son entendidos, bajo el hecho de que algunos polisacáridos en concentraciones bajas disminuyen su viscosidad, cuando se encuentran bajo la influencia de alguna sal [20].

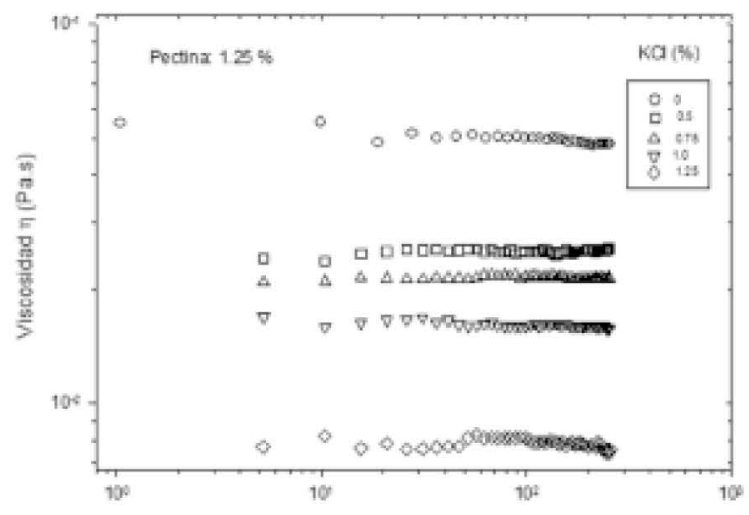

Figura 7. Reograma de una solución de pectina al 1.25 $\%$ con $\mathrm{KCl}$ a diferentes concentraciones.

Por otra parte, el efecto de la adición de isopropanol a las soluciones de pectina, se presenta en la Figura 8. Esta perturbación provoca un cambio significativo en su comportamiento reológico, al pasar de ser un fluido newtoniano a uno no-Newtoniano de adelgazamiento a la cizalla, a concentraciones mayores a $20 \% \mathrm{v} / \mathrm{v}$. Aunque a una concentración de $10 \% \mathrm{v} / \mathrm{v}$, la respuesta al incremento de la velocidad de corte, se observa un comportamiento similar al caso de adición de $\mathrm{KCl}$. 


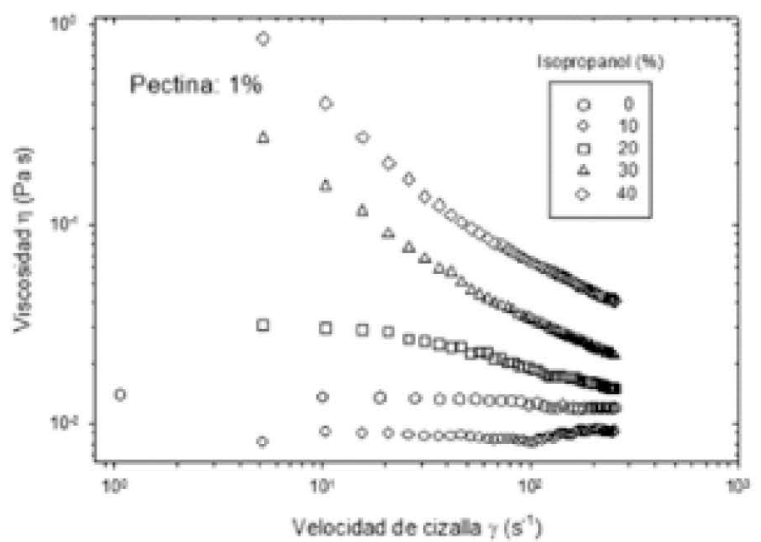

Figura 8. Reograma de una solución de pectina al $1 \%$ con Isopropanol a diferentes concentraciones.

Los resultados presentes en los reogramas de la Figura 8, sugieren que la molécula de pectina tiende a compactarse por la presencia del alcohol [12]. Es preciso mencionar que, las concentraciones empleadas para precipitar y separar la pectina dependen de la concentración del polímero. En consecuencia, es de esperarse que a medida que la concentración de isopropanol aumente, la molécula tiende a cambiar su conformación a una más compacta y precipita a mayores concentraciones de alcohol [21].

En un intento por encontrar algún efecto diferente a los anteriores, para mejorar los valores en el flux durante la ultrafiltración, se estudió la respuesta a la adición simultánea de isopropanol y $\mathrm{KCl}$. Los resultados de esta perturbación combinada de compuestos, tuvieron mayores efectos en la reología de las disoluciones. El efecto resultante, al combinar los 3 componentes se vuelve más evidente que con las situaciones de perturbaciones anteriores, alcanzando en la mayoría de los casos la aparición de un precipitado tipo gel y por lo tanto una disolución no homogénea, esto impiden una correcta medición de la viscosidad.

\subsection{Efectos en la Ultrafiltración}

El flux volumétrico durante la ultrafiltración de las soluciones de pectina a diferentes concentraciones se presenta en la Figura 9, en ella se observa una disminución en el mismo al aumentar la concentración del polímero. El estado estable de permeado en la membrana se alcanza en tiempos mayores a los 100 minutos, lo que representa una etapa de transición larga en comparación con el flux de permeado obtenido.

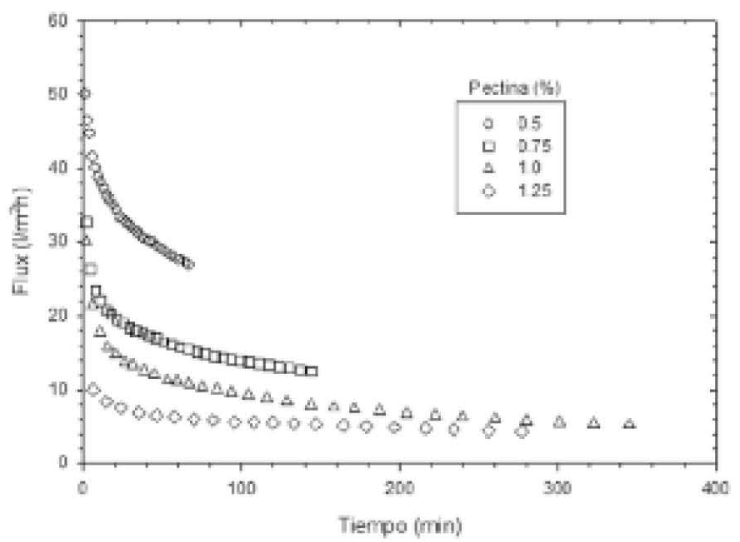

Figura 9. Efecto de la concentración de pectina en el flux volumétrico durante el proceso de UF.

El tiempo para alcanzar el estado estable reportado por algunos autores en soluciones de pectina [18, 22], varían entre los 10 y 40 minutos. En estos estudios, tenían condiciones de operación diferentes, resaltando las mayores velocidades tangenciales al interior de la membrana, esta situación favorece el permeado de la solución, al propiciar menor acumulación de polisacárido sobre su superficie debido al efecto de arrastre causado por la velocidad. Otro aspecto importante en la disminución del tiempo de permeado, es el tipo de empaque de la membrana, en las reportadas en la literatura, los fabricantes aseguran que retienen como mínimo el $90 \%$ de las partículas que están comprendidas en el tamaño que aseguran no pasan través de la misma y en nuestro caso, el fabricante, asegura que el $99 \%$ de las partículas son retenidas, en consecuencia se tiene un permeado con menor concentración de polisacárido. Esta diferencia significa un menor efecto de taponamiento sobre su superficie, cuando se tienen tamaños de poro más grandes. Esta característica facilita el fenómeno de polarización sobre la superficie de la membrana y dificulta el proceso de filtrado a través de la misma.

\subsection{Efectos de la velocidad axial}

La velocidad axial sobre la superficie de la membrana de ultrafiltración fue estudiada en este trabajo (Figura 10), estas fueron elegidas en función de las recomendadas por el fabricante. Los experimentos se llevaron a cabo bajo un régimen de flujo laminar y con un flux en estado estable. Los resultados se representan con un análisis de regresión lineal y se resumen en la Tabla 3. 


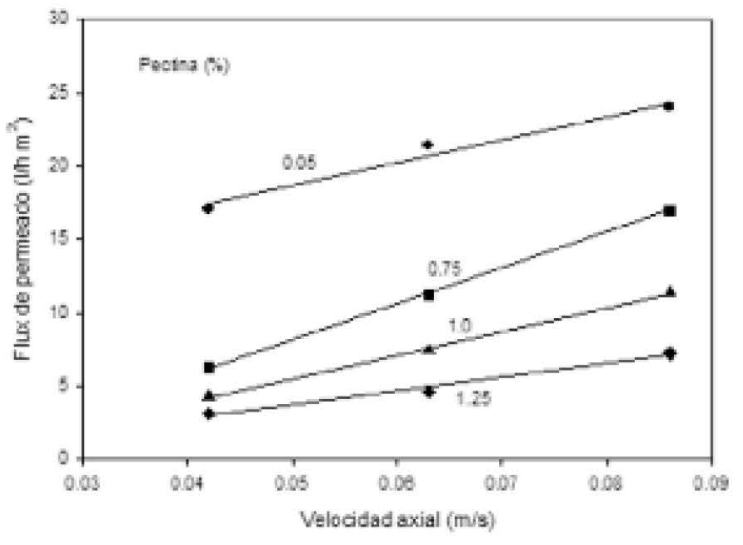

Figura 10. Efecto de la velocidad axial en la ultrafiltración de soluciones de pectina. Las líneas continuas representan los resultados del análisis de regresión lineal.

La fórmula empleada para obtener los valores de la regresión lineal es:

$$
f l u x=h_{0}+A \cdot \text { Concentración }
$$

donde: $\mathrm{h}_{0}=$ Constante para cada concentración, $\mathrm{A}=$ Pendiente de la velocidad axial, $\mathrm{V}_{\min }=$ Velocidad mínima de operación, $\mathrm{V}_{\max }=$ Velocidad máxima de operación.

Tabla 3. Datos de la regresión lineal a diferentes velocidades axiales en soluciones de pectina.

\begin{tabular}{ccccc}
\hline Concentración & $\mathbf{h}_{\mathbf{0}}$ & $\mathbf{A}$ & $\mathbf{V}_{\min }$ & $\mathbf{V}_{\max }$ \\
\hline $0.5 \%$ & 10.81 & 157.0 & 0.042 & 0.086 \\
$0.75 \%$ & -4.18 & 246.0 & 0.042 & 0.086 \\
$1.0 \%$ & -2.65 & 162.5 & 0.042 & 0.086 \\
$1.25 \%$ & -0.99 & 93.5 & 0.042 & 0.086 \\
\hline
\end{tabular}

A partir de los datos reportados en la Tabla 3, es posible estimar el flux de permeado para soluciones de pectina, cuyas velocidades de operación y concentración de las soluciones estén comprendidas entre los rangos estudiados. No es recomendable extrapolar datos, debido a que no se puede inferir el comportamiento de las soluciones fuera de los mismos. Con estos resultados se confirma que a mayores velocidades axiales de operación, se obtienen mayores flujos de permeado, lo que concuerda con resultados reportados en la literatura [23], en el cual proponen un modelo para predecir el flux de soluciones de fluidos no newtonianos y donde la velocidad axial de operación es una de las variables que afectan el flux de las soluciones que se sometan a un proceso de ultrafiltración.

\subsection{Efecto de la perturbación de las soluciones con KCl e Isopropanol}

En la Figura 11 se observan curvas típicas de $(\mathrm{t} / \mathrm{Vp})$ contra $\mathrm{Vp}$ para el cálculo de la resistencia específica de la membrana $\left(\propto_{P}\right)$. El valor de la pendiente es el que permite realizar el cálculo de $\propto_{P}$ y dicho valor es diferente para soluciones con concentraciones diferentes. A partir de la regresión lineal, se obtiene la pendiente y la constante, el valor de la pendiente es igual a $\alpha_{P} C_{B} \mu / A^{2} \Delta P\left(\mathrm{~s} \cdot \mathrm{m}^{-6}\right)$. Sustituyendo los datos conocidos de concentración $\left(C_{B}\right)$, viscosidad $(\mu)$, área de transferencia de masa de la membrana $(A)$ y la presión $(\Delta P)$, se despeja $\alpha_{P}$ y se obtiene su valor. En la Tabla 4 se presentan los valores de los coeficientes obtenidos mediante regresión de los datos de la Figura 11.

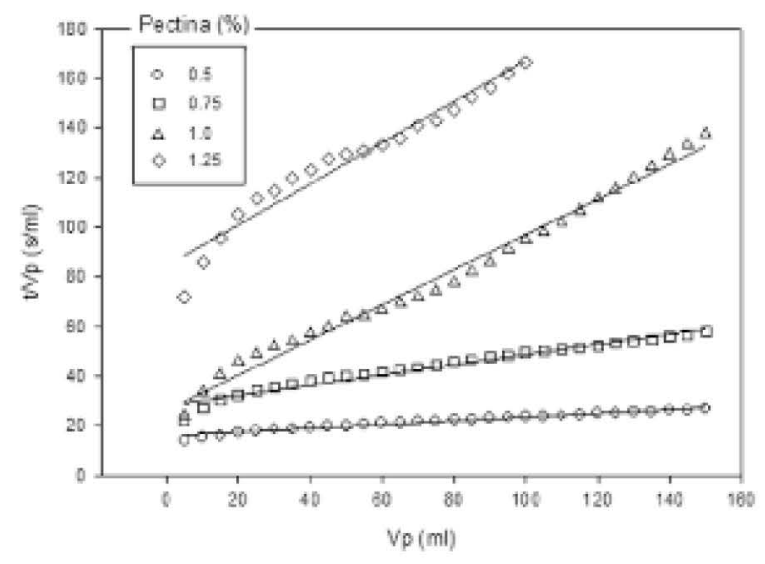

Figura 11. Curvas típicas de (t/VP) contra Vp obtenidas de la ultrafiltración de soluciones de pectina.

Tabla 4. Datos obtenidos de la regresión lineal realizada a los valores de la Figura 11 a diferentes concentraciones de pectina.

\begin{tabular}{ccccc}
\hline \multirow{2}{*}{ Coeficientes } & \multicolumn{4}{c}{ Concentración de Pectina } \\
\cline { 2 - 5 } & $\mathbf{0 . 5}$ & $\mathbf{0 . 7 5}$ & $\mathbf{1 . 0}$ & $\mathbf{1 . 2 5}$ \\
\hline$\mu \mathrm{R}_{\mathrm{m}} / \Delta \mathrm{PA}\left(\mathrm{s} \cdot \mathrm{m}^{-3}\right)$ & 15.87 & 28.31 & 26.42 & 84.33 \\
& 0.08 & 0.21 & 0.71 & 0.83 \\
$\left(\mathrm{~s} \cdot \mathrm{m}^{-6}\right)$ & 0.97 & 0.96 & 0.99 & 0.95 \\
$\mathrm{r}^{2}$ & & & & \\
\hline
\end{tabular}

Cuando se perturban las soluciones con $\mathrm{KCl}$, se observa un incremento significativo en el valor de $\propto_{P}$ (Tabla 5), por lo que se obtienen valores de flux de permeado más bajos que los obtenidos sin 
agregar la sal. Efectos similares fueron observados en la ultrafiltración de soluciones de xantana tratadas con esta sal [7]. Estos resultados sugieren que el mayor ordenamiento de las moléculas causado por la presencia de perturbaciones iónicas, afecta significativamente la estructura de la capa de polarización depositada sobre la membrana durante la ultrafiltración, lo que coincide con resultados reportados en el tratamiento de pectina con diferentes concentraciones de glucosa [22]. La adición de $\mathrm{KCl}$ en la solución acarrea dos efectos; el primero, la disminución en la viscosidad de la solución (Figura 7) y, el segundo, un incremento en la $\propto_{P}$ de las disoluciones, siendo este efecto, es el que reduce directamente su flux de permeado, aumentando el tiempo de operación de la ultrafiltración.

Tabla 5. Resistencia especifica de la membrana y flux volumétrico obtenido durante la ultrafiltración de soluciones de pectina conteniendo $\mathrm{KCl}$.

\begin{tabular}{|c|c|c|c|c|}
\hline $\begin{array}{l}\text { Pectina } \\
(\% \mathrm{p} / \mathrm{V} \\
\left.\mathrm{H}_{2} \mathrm{O}\right) \\
\end{array}$ & $\begin{array}{c}\mathrm{KCl} \\
(\% \mathrm{p} / \mathrm{V} \\
\left.\mathrm{H}_{2} \mathrm{O}\right) \\
\end{array}$ & $\begin{array}{c}\text { Resistencia } \\
\text { específica } \alpha_{\mathrm{P}} \mathrm{e}-3 \\
(\mathrm{Kg} \cdot \mathrm{m})^{-1} \\
\end{array}$ & $\begin{array}{c}\text { Flux } \\
\text { volumétrico } \\
\text { cte. }\left(1 / \mathrm{m}^{2}-\mathrm{h}\right) \\
\end{array}$ & $\begin{array}{c}\text { Estado } \\
\text { físico de } \\
\text { la solución } \\
\end{array}$ \\
\hline 0.5 & -- & 3.49 & 21.37 & I \\
\hline 0.75 & --- & 2.1022 & 11.23 & I \\
\hline 1.0 & --- & 3.2540 & 7.4 & I \\
\hline 1.25 & --- & 1.0120 & 4.6 & I \\
\hline 0.5 & 0.5 & 21.4 & 14.79 & I \\
\hline 0.5 & 0.75 & 29.23 & 11.94 & I \\
\hline 0.5 & 1.0 & 73.21 & 10.25 & I \\
\hline 0.5 & 1.25 & 54.08 & 9.66 & I \\
\hline 0.75 & 0.5 & 22.15 & 7.15 & I \\
\hline 0.75 & 0.75 & 39.64 & 6.27 & I \\
\hline 0.75 & 1.0 & 122.7 & 5.65 & I \\
\hline 0.75 & 1.25 & 295.7 & 4.6 & $\mathrm{I}$ \\
\hline 1.0 & 0.5 & 24.28 & 6.6 & I \\
\hline 1.0 & 0.75 & 20.18 & 6.1 & I \\
\hline 1.0 & 1.0 & 35.52 & 5.85 & I \\
\hline 1.0 & 1.25 & 59.06 & 5.33 & $\mathrm{I}$ \\
\hline 1.25 & 0.5 & 15.38 & 3.99 & I \\
\hline 1.25 & 0.75 & 29.78 & 2.94 & I \\
\hline 1.25 & 1.0 & 97.99 & 2.43 & I \\
\hline
\end{tabular}

Cuando se perturba a las muestras con la adición de un solvente, se obtienen valores de flux de permeado menores a los alcanzados sin ninguna perturbación. Asimismo, el valor de $\propto_{P}$ para concentraciones de pectina menores a $1 \%$, disminuye conforme la concentración de pectina e isopropanol aumenta (Tabla 6); aunque, para concentraciones mayores a $1 \%$ de soluto, $\propto_{P}$ aumenta al ir incrementar la concentración de alcohol. Se puede observar que la resistencia específica de la membrana con una solución de pectina al $0.5 \% \mathrm{p} / \mathrm{v}$ e isopropanol al $20 \% \mathrm{v} / \mathrm{v}$, es casi 14 veces mayor que a $0 \% \mathrm{v} / \mathrm{v}$, y esta a su vez es casi 4 veces mayor que a una concentración de $50 \% \mathrm{v} / \mathrm{v}$ de alcohol. Este efecto disminuye progresivamente al aumentar la concentración del polisacárido (Tabla 6). De lo anterior, es posible determinar que a medida que se intensifica la concentración de isopropanol en las soluciones, se obtiene una disminución en el flux de permeado de ultrafiltración. Sin embargo, aunque la $\propto_{P}$ disminuye en muchos casos, esto no se refleja en un aumento en el flux de permeado.

Tabla 6. Resistencia especifica de la membrana y flux volumétrico obtenido durante la ultrafiltración de soluciones de pectina conteniendo Isopropanol.

\begin{tabular}{|c|c|c|c|c|}
\hline $\begin{array}{c}\text { Pectina } \\
(\% \mathrm{p} / \mathrm{V} \\
\left.\mathrm{H}_{2} \mathrm{O}\right)\end{array}$ & $\begin{array}{c}\text { Isopro- } \\
\text { panol } \\
(\% \mathrm{~V} / \mathrm{V})\end{array}$ & $\begin{array}{c}\text { Resistencia } \\
\text { específica } \\
\alpha_{\mathrm{P}} \mathrm{e}-3 \\
(\mathrm{Kg} \cdot \mathrm{m})^{-1}\end{array}$ & $\begin{array}{c}\text { Flux } \\
\text { volumétrico } \\
\text { cte. }\left(\mathrm{l} / \mathrm{m}^{2}-\mathrm{h}\right)\end{array}$ & $\begin{array}{c}\text { Estado } \\
\text { físico de } \\
\text { la } \\
\text { solución }\end{array}$ \\
\hline 0.5 & --- & 3.49 & 21.4 & I \\
\hline 0.75 & --- & 2.10 & 11.2 & I \\
\hline 1.0 & --- & 3.25 & 7.4 & I \\
\hline 1.25 & --- & 1.01 & 4.6 & I \\
\hline 0.5 & 20 & 48.8 & 9.9 & I \\
\hline 0.5 & 30 & 20.5 & 9.9 & I \\
\hline 0.5 & 40 & 15.2 & 9.5 & I \\
\hline 0.5 & 50 & 13.4 & 9.4 & I \\
\hline 0.75 & 20 & 7.23 & 8.7 & I \\
\hline 0.75 & 30 & 5.81 & 7.8 & II \\
\hline 0.75 & 40 & 4.43 & 6.4 & II \\
\hline 0.75 & 50 & 2.43 & 6.2 & II \\
\hline 1.0 & 20 & 4.43 & 7.4 & I \\
\hline 1.0 & 30 & 8.56 & 6.5 & II \\
\hline 1.25 & 20 & 3.84 & 4.5 & I \\
\hline 1.25 & 30 & 4.43 & 3.7 & II \\
\hline
\end{tabular}

Las soluciones conteniendo isopropanol presentaron una apariencia viscosa y de gel (estados físicos I - IV, ver F igura 2). Los mismos efectos se encontraron en estudios reportados en la literatura para las soluciones de pectina con etanol [12]. Por lo tanto, la caída adicional en el flux de la membrana cuando se procesan las soluciones con estos solventes indica que la capa de polarización formada en la superficie de la membrana posee una estructura de gel, la cual favorece una mayor resistencia al flux. Resultados como estos, fueron reportados para goma xantana [9]. 
Tabla 7. Resistencia especifica de la membrana y flux volumétrico obtenido durante la ultrafiltración de soluciones de pectina conteniendo Isopropanol.

\begin{tabular}{|c|c|c|c|c|c|}
\hline $\begin{array}{c}\text { Pectina } \\
\left(\% \text { p/v } \mathbf{H}_{2} \mathrm{O}\right)\end{array}$ & 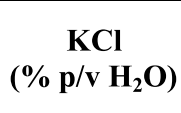 & $\begin{array}{c}\text { Isopropanol } \\
(\% \mathrm{v} / \mathrm{v})\end{array}$ & $\begin{array}{c}\text { Resistencia } \\
\text { específica } \alpha_{P} \text { e-3 } \\
(\mathrm{Kgm})^{-1}\end{array}$ & $\begin{array}{c}\text { Flux volumétrico } \\
\text { cte. }\left(1 / \mathbf{m}^{2} \mathbf{h}\right)\end{array}$ & $\begin{array}{c}\text { Estado } \\
\text { físico de la } \\
\text { solución }\end{array}$ \\
\hline 0.5 & 0.5 & 20 & 16.3 & 22.6 & I \\
\hline 0.5 & 0.5 & 30 & 25.1 & 10.3 & I \\
\hline 0.5 & 0.75 & 20 & 10.7 & 10.0 & I \\
\hline 0.5 & 1.0 & 20 & 6.8 & 9.9 & I \\
\hline 0.5 & 1.25 & 20 & 0.48 & 9.8 & I \\
\hline 0.75 & 0.5 & 20 & 1.9 & 7.9 & II \\
\hline 0.75 & 1.0 & 20 & 1.0 & 6.1 & III \\
\hline 0.75 & 1.0 & 30 & 0.68 & 5.8 & II \\
\hline 1.0 & 1.0 & 20 & 1.2 & 5.9 & $\mathrm{~V}$ \\
\hline
\end{tabular}

A partir de estos resultados puede decirse que la adición tanto de $\mathrm{KCl}$ como de isopropanol, inducen cambios estructurales en la pectina en solución, estos cambios aumentan la resistencia de la capa de polarización formada en la membrana de ultrafiltración.

El efecto combinado de la adición de $\mathrm{KCl}$ e isopropanol en la estructura de la pectina en solución, en términos del impacto en la resistencia específica de la membrana y del rendimiento obtenido en la ultrafiltración, se presenta en la Tabla 7. En ésta, se puede observar que la combinación de los solutos adicionados bajo las condiciones estudiadas, produce en algunos casos valores de $\propto_{P}$ menores a los que se presentan con soluciones de pectina sin ningún pretratamiento. Sin embargo, aun así, debido a la tendencia de la naturaleza de la pectina a formar geles fácilmente, el flux de permeado disminuye, afectando de forma negativa el proceso. En algunos casos, durante la operación de ultrafiltración y después de tratar el $10 \%$ de la solución problema, se producen problemas de taponamiento de la membrana debido a la formación de un gel, bloqueando casi completamente el paso de la solución a través de la membrana. Como una consecuencia de lo anterior, los valores en el flux de permeado, fueron extremadamente bajos, esto se aprecia en la Tabla 7.

Estos resultados indican que el precipitado tipo gel que se forman cuando se combinan bajas concentraciones de $\mathrm{KCl}$ y de isopropanol (producto de una transición en la solubilidad debido a cambios en la afinidad polímero-solvente) presentan en general la mayor resistencia al flux al ser depositados en la superficie de la membrana. Similares resultados fueron obtenidos para soluciones de xantana perturbadas con $\mathrm{KCl}$ e isopropanol [10] y para gelatina [15], quienes observaron que las morfologías de agregados densos, suaves tipo gel, formaron películas de polarización de alta resistencia al flux. En su estudio reporta un incremento en $\propto_{P}$ y una disminución en el flux de las soluciones. Los datos experimentales presentados en la Tabla 7, muestran que, para la combinación de $0.5 \%$ pectina, $0.5 \%$ $\mathrm{KCl}$ y $20 \%$ isopropanol, alcanza un flux volumétrico de $22.6 \mathrm{l} / \mathrm{m}^{2} \mathrm{~h}$ y para las soluciones de pectina sola de $21.4 \mathrm{1} / \mathrm{m}^{2} \mathrm{~h}$, lo que representa un aumento en términos del flux de $5 \%$, pero la resistencia específica de la membrana aumenta casi en 5 veces su valor, lo que reduce su período de vida. El aumento en el flux es pequeño, si se compara con el gasto de adicionar $\mathrm{KCl}$ e isopropanol a las soluciones de pectina al purificarlo nuevamente para su uso.

\subsection{Efecto de la Ultrafiltración en la reología de las soluciones}

El efecto que provoca en la reología de las soluciones de pectina tratadas con Ultrafiltración se muestra en la Figura 12. En este caso, se observa que una disolución concentrada del $1 \%$ p/v al $2 \%$ $\mathrm{p} / \mathrm{v}$ cambia su comportamiento reológico de fluido Newtoniano a no-Newtoniano de adelgazamiento a la cizalla. Este resultado pone de manifiesto que esta operación de separación provoca una compactación en la molécula de la pectina en solución, lo que afecta significativamente su respuesta al ser sometida a esfuerzos de corte.

Este resultado pone de manifiesto la necesidad de estudiar a profundidad este comportamiento y las causas que lo originan.

\section{Conclusiones}

La adición de $\mathrm{KCl}$ y/o isopropanol a la pectina en solución ocasionó la aparición de 6 diferentes morfologías en las soluciones, derivadas del análisis visual; estos cambios estructurales dan como respuesta un cambio en su comportamiento 


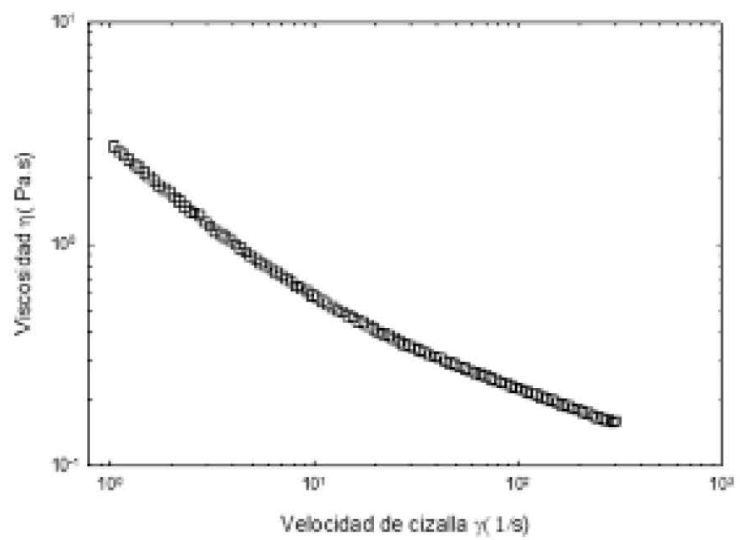

Figura 12. Reograma del concentrado de solución de pectina del 1 al $2 \%$.

reológico, obteniendo disminución de la viscosidad para el caso de adición de la sal $\mathrm{y}$, para la incorporación de isopropanol se observó un cambio de fluido Newtoniano a no-Newtoniano de adelgazamiento a la cizalla. El flux obtenido durante la operación de ultrafiltración decrece al perturbar las soluciones con estos compuestos, así mismo, se encontró que la resistencia especifica de la membrana aumenta y en consecuencia se reduce su período de vida útil. Además, al realizar pruebas de reología de cizalla simple a las soluciones concentradas mediante ultrafiltración, se obtiene una respuesta de fluido no-Newtoniano, a concentraciones que al prepararlas de la forma descrita en el desarrollo experimental presentan una respuesta Newtoniana, este fenomenología detectada, da pie a un estudio más profundo para entender el origen de este cambio. Finalmente, se determinó que las técnicas estudiadas en el presente trabajo, para favorecer el proceso de ultrafiltración de las soluciones de Pectina no son adecuadas, debido a que no se incrementa el flux a través de la membrana y por el contrario, incrementan su taponamiento en períodos cortos de operación.

\section{Referencias}

1. Parvatiyar, M. J. Membr. Sci. 1998, 148 45-57.

2. Agashichev, S. P.; Falalejev, D. V. J. Membr. Sci. 2000, 171, 173-178.

3. Belfort, G.; Davis R.; Sydney A. J. Membr Sci. 1994, 96, 158.

4. Curcio, S.; Calabro, V.; Iorio, G.; Cindio b. J. Food Eng. 2000, 48, 235-241.

5. Bacchin, P.; Hassen, D.S.; Starov, V.; Clifton, M. J.; Aimar, P. Chem. Eng. Sci. 2002, 57, 77-91.

6. Perry, R.; Green, D.; Maloney, J. Manual del Ingeniero Quimico. 6ta. ed. Mc Graw Hill, México 1992, 17.16 17.32.

7. Rield, K.; Girard, B.; Lencki, R. J. Membr. Sci. 1998, 139, 155-166.
8. Noordman, T. R.; Ketelaar, T. H.; Donkers, F.; Wesselingh, J. A. 2002, 57, 693-703.

9. Torrestiana-Sanchez, B.; Balderas-Luna, L.; Brito, E.; Lencki, W. R. J. Membr. Sci. 2007, 294, 84-92.

10. Hernández-Campos, F. J.; Brito-De La Fuente, E.; Torrestiana-Sanchez, B. J. Agric. Food Chem. 2010, 58, 187-193.

11. Gulfi, M.; Arrigoni, E.; Amado, R. Carbohydr. Polym. 2004, 20, 1-9.

12. Cho, W. CH.; Lee, Y. Y.; Kim, W. CH. Carbohydr. Polym. 2003, 54, 21-26.

13. Leroux, J.; Langendorff, V.; Schick, G.; Vaishnav, V.; Mazoyer, J. Food Hydrocolloids, 2003, 17, 455-462.

14. Kuuva, T.; Lantto, R.; Reinikainen, T.; Buchert, J.; Autio, K. Food Hydrocolloids, 2003, 17, 679-684.

15. Elysee-Collen, B.; Lencki, R. W. J. Agric. Food Chem. 1996a, 44, 1651-1657.

16. Evageliou, V.; Ptitchkina, N. M.; Morris E. R. Food Hydrocolloids. 2005, 20, 1-5.

17. Donato, L.; Garnier, C.; Novales, B.; Doublier, J. L. Food Hydrocolloids, 2005, 19, 549-556.

18. Charcosset, C.; Choplin, L. J. Membr. Sci. 1996, 115, 147160.

19. Pritchard, M.; Howell, J.; Wield, R. J. Membr. Sci. 1995, 102, 223-235.

20. Mesbahi, G.; Jamalian, J.; Farahnaky, A. Food Hydrocolloids. 2005, 19, 731-738.

21. Arsalan, N. J. Food Sci. Journal of Food Science and Technology, 1995, 32, 381-385.

22. Sulaiman, Z. M.; Sulaiman, M. N.; Shamel, M.. Chemical Engineering Journal, 2001, 84, 557-563.

23. Agashichev, S. P.; Falalejev, D. V. J. Membr. Sci. 2000, 171, 173-178. 